\title{
Awareness of Prediabetes Status and Subsequent Health Behavior, Body Weight, and Blood Glucose Levels
}

\author{
Ibiye Owei, MD, MPH, Nkiru Umekwe, MBBS, Fatoumatta Ceesay, BS, \\ and Samuel Dagogo-Jack, MD
}

Background: Lifestyle intervention decreases diabetes risk in prediabetic subjects, but the impact of passive notification of prediabetes status on glycemia or health behavior is unclear.

Methods: The Pathobiology of Prediabetes in a Biracial Cohort (POP-ABC) study followed normoglycemic African American (AA) and European American (EA) offspring of parents with type 2 diabetes mellitus for incident prediabetes. During 5.5 years of follow-up (mean, 2.62 years), 101 of 343 subjects developed prediabetes and were notified, without any interventions. Participants were recalled 18 months poststudy. Here, we compared data from participants with incident prediabetes or normoglycemia (control) during POP-ABC who underwent retesting 18-months poststudy.

Results: There were 73 subjects ( 46 female, 27 male; $36 \mathrm{AA}, 37 \mathrm{EA}$ ) in the prediabetes group and 73 subjects ( 48 female, 25 male; $35 \mathrm{AA}, 38 \mathrm{EA}$ ) in the control group. The mean ( \pm SEM) enrollment age was $48.7 \pm 0.96$ years versus $48.3 \pm 1.06$ years $(P=.37)$ and body mass index $(B M I)$ was $31.1 \pm 0.70$ $\mathrm{kg} / \mathrm{m}^{2}$ versus $29.2 \pm 0.69 \mathrm{~kg} / \mathrm{m}^{2}(P=.04)$ for prediabetes versus control groups, respectively. The 18-month changes (prediabetes vs control) were the following: fasting plasma glucose (FPG), $-8.01 \pm$ 1.11 vs $2.02 \pm 0.64 \mathrm{mg} / \mathrm{dL}$; 2-hour plasma glucose $(2 \mathrm{hrPG}),-8.21 \pm 3.34 \mathrm{vs} 8.53 \pm 3.17 \mathrm{mg} / \mathrm{dL}$; weight, $-0.54 \pm 0.72$ vs $2.77 \pm 1.25 \mathrm{~kg}$; and waist circumference $-1.07 \pm 0.78$ vs $1.78 \pm 0.85(P=$ $.03-<0.0001)$. The interval changes in FPG were significantly correlated with changes in weight and waist circumference $(r=0.2, P=.01)$. The prediabetes group reported improved dietary and exercise habits compared with control.

Conclusion: Communication of prediabetes status is associated with improvements in glucose tolerance, glycemia, and adiposity, probably via self-directed lifestyle modification. (J Am Board Fam Med 2019;32:20-27.)

Keywords: Health Behavior, Life Style, Prediabetes

The Department of Health and Human Services (HHS) and the American Diabetes Association (ADA) drew attention to prediabetes at a joint press

This article was externally peer reviewed.

Submitted 21 August 2018; revised 27 September 2018; accepted 2 October 2018.

From Department of Medicine, Division of Endocrinology, Diabetes and Metabolism, University of Tennessee Health Science Center, Memphis, TN.

Funding: This study was supported by Grant R01 DK067269 from the National Institutes of Health and Grant 7-07-MN-13 from the ADA, both awarded to SD-J. The funding sources had no role in the design and execution of the study, or analysis and publication of the data obtained from the study.

Conflict of interest: none declared.

Corresponding author: Sam Dagogo-Jack, MD, University of Tennessee Health Science Center, 920 Madison Avenue, Memphis, Tennessee 38163 (E-mail: sdj@uthsc.edu). conference: "HHS and the ADA are using the new term 'prediabetes' to describe an increasingly common condition in which blood glucose levels are higher than normal but not yet diabetic-known in medicine as impaired glucose tolerance (IGT) or impaired fasting glucose (IFG). Studies have shown that most people with this condition go on to develop type 2 diabetes within 10 years." ${ }^{\text {T }}$ The Centers for Disease Control and Prevention estimates that 84.1 million US adults aged 18 years or older ( $~ 34 \%$ of the adult US population) had prediabetes in $2015 .^{2}$ Worldwide, more than 300 million people are estimated to have prediabetes. ${ }^{3}$

The diagnosis of prediabetes is established by the documentation of IFG, indicated by a fasting plasma glucose (FPG) level of $100 \mathrm{mg} / \mathrm{dL}$ to 125 
$\mathrm{mg} / \mathrm{dL}(5.6-6.9 \mathrm{mmol} / \mathrm{L})$ or IGT, indicated by a 2-hour plasma glucose (2hrPG) level of $140 \mathrm{mg} / \mathrm{dL}$ to $199 \mathrm{mg} / \mathrm{dL}(7.8-11.0 \mathrm{mmol} / \mathrm{L})$ during a $75-\mathrm{g}$ oral glucose tolerance test (OGTT). ${ }^{4,5}$ Hemoglobin A1c (HbA1c) levels of 5.7-6.4\% are also diagnostic of prediabetes. ${ }^{4}$ Besides the risk of developing type 2 diabetes mellitus (T2DM), people with prediabetes are at increased risks for heart disease, stroke, neuropathy, and other microvascular complications. ${ }^{6-8}$

Several landmark clinical trials have demonstrated that lifestyle modification decreases blood glucose levels and prevents progression to T2DM in persons with prevalent prediabetes. ${ }^{9,12}$ In those studies, teams of dietitians, exercise physiologists, and other clinicians executed the standard lifestyle intervention protocols that led to the improvement in glucose tolerance and successful prevention of T2DM in study participants. ${ }^{9,11,13}$ Compared with the placebo group, individuals assigned to lifestyle intervention experienced $40-58 \%$ relative reduction in the risk of developing T2DM. ${ }^{9,11}$ Notably, the participants in the landmark diabetes prevention studies all had prevalent prediabetes determined during cross-sectional population screening and, therefore, of unknown duration at enrollment. $^{9-11}$

The impact of awareness of incident prediabetes status on glycemic trajectories among previously normoglycemic persons is unknown, and cross-sectional surveys show conflicting reports regarding the impact of such awareness on self-directed health behavior. ${ }^{14,15}$ The Pathobiology of Prediabetes in A Biracial Cohort (POP-ABC) ${ }^{16,17}$ study enrolled normoglycemic African American (AA) and European American (EA) offspring of parents with T2DM and followed them for the occurrence of incident prediabetes, defined as IFG and/or IGT. ${ }^{4,5}$ All POP-ABC study participants completed a screening OGTT before enrollment. The first subject was enrolled in September 2006, and the POP-ABC study ended in February 2012 after the final follow-up visits. During $\sim 5$ years of follow-up, 101 of 343 subjects developed prediabetes and were duly notified, without any active intervention or counseling, as POP-ABC was a natural history study.

Eighteen months after the study ended, POP$\mathrm{ABC}$ study participants were invited to return for OGTT and clinical examination, as part of the screening procedures for a newly funded Pathobi- ology and Reversibility of Prediabetes in A Biracial Cohort (PROP-ABC) study, whose aims include intensive lifestyle intervention to reverse prediabetes (ClinicalTrials.gov Identifier NCT02027571). Here, we report temporal changes in glucose and other metabolic measures between the end of POP$\mathrm{ABC}$ and the beginning of PROP-ABC (18 months later) in participants with incident prediabetes during POP-ABC (pre-diabetes mellitus group) compared with a matched group of POP-ABC participants who did not develop prediabetes.

\section{Methods \\ Study Subjects}

The study subjects were participants in the POPABC study. ${ }^{16,17}$ Eligibility criteria for the POPABC study included age 18 to 65 years, self-reported non-Hispanic white (EA) or non-Hispanic black (AA) race/ethnicity status; 1 or both biological ]parents with T2DM; no evidence of diabetes; normal FPG $(<100 \mathrm{mg} / \mathrm{dL}[<5.6 \mathrm{mmol} / \mathrm{L}])$ and/or normal $2 \mathrm{hrPG}(<140 \mathrm{mg} / \mathrm{dL}[<7.8 \mathrm{mmol} / \mathrm{L}])$ during a 75-g OGTT; and good overall health, as previously described. ${ }^{16,17}$ None of the participants was using any medications known to alter glucose or lipid metabolism. Enrollment in behavioral, pharmacologic, or other active weight loss program or a history of liposuction or bariatric surgery were additional exclusion criteria. ${ }^{16,17}$ The primary outcome was the occurrence of prediabetes (IFG and/or IGT) or diabetes, defined by the ADA criteria. ${ }^{4}$ A confirmatory test was performed within 6 weeks of each endpoint occurrence. The 75-g OGTT was the method of confirmation. All endpoints were independently adjudicated by the Institutional Data and Safety Officer (Murray Heimberg, $\mathrm{MD}, \mathrm{PhD})$. The study protocols were approved by the University of Tennessee Institutional Review Board; all participants gave written informed consent before initiation of study procedures.

\section{Chronology and Outcome}

The first subject was enrolled in September 2006 and the POP-ABC study ended in February 2012 after the final follow-up visits. ${ }^{16,17}$ During 5.5 years of follow-up (mean, 2.62 years), 101 of 343 participants developed incident prediabetes. ${ }^{17}$ Per the study protocol, a copy of the confirmatory OGTT result was provided to all participants reaching the 
Figure 1. Study design and time intervals of data collection. Prediabetes outcome was assessed after $\sim 5$ years of follow-up of initially normoglycemic POP-ABC participants. Eighteen months later, POP-ABC study participants were rescreened for a newly funded extension PROP-ABC study. The present report compares the 18-month interval changes in glycemic and other measurements between participants who developed prediabetes during POP-ABC study and those who remained normoglycemic.

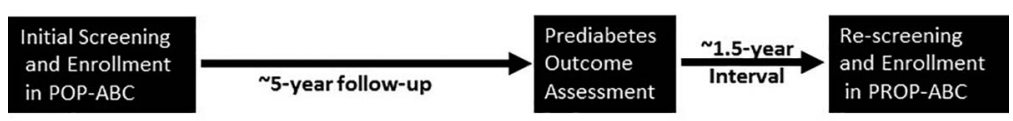

POP-ABC, Pathobiology of Prediabetes in A Biracial Cohort study

PROP-ABC, Pathobiology and Reversibility of Pre diabetes in A Biracial Cohort study

endpoint. However, no intervention or counseling was provided, as $\mathrm{POP}-\mathrm{ABC}$ was a purely natural history study. In September 2013, 18 months after closeout of the POP-ABC study, funding became available for a continuation study, the PROP-ABC, whose goal is to provide intensive lifestyle intervention to the POP-ABC participants with incident prediabetes (ClinicalTrials.gov Identifier NCT02027571). Eligibility for PROP-ABC thus was limited to the original POP-ABC participants, all of whom were invited to join the new study. Interested participants underwent a standard OGTT, anthropometry, and clinical examination, as part of the baseline reenrollment procedures. To test the hypothesis that awareness of incident prediabetes status triggers self-directed health behaviors that improve metabolic endpoints, we compared the temporal trends in OGTT, anthropometric, and lipid data between the end of POP$\mathrm{ABC}$ and the initiation of PROP-ABC study in participants with incident prediabetes during the POP$\mathrm{ABC}$ phase and an age-, sex-, and ethnicity-matched control group. We also compared changes in selfreported dietary and physical activity behaviors between the 2 groups of participants.

Figure 1 summarizes the time intervals from the beginning until the end of the POP-ABC study to rescreening for the extension PROP-ABC study.

\section{Assessments}

Participants arrived at the University of Tennessee General Clinical Research Center after an overnight fast to undergo physical examination and scheduled assessments. Height was recorded using a stadiometer, weight was measured with a digital scale (Tanita WB-300 Plus Digital, Arlington Heights, IL), and body mass index (BMI) was calculated as weight $(\mathrm{kg})$ divided by the square of the height in meters. A Gulick II tape was used to measure waist circumference, as previously described. ${ }^{16,17}$ Usual food intake was captured using the Food Habits Questionnaire (FHQ) ${ }^{18}$ and habitual physical activity was recorded using the Modifiable Activity Questionnaire (MAQ), ${ }^{19}$ as previously described..$^{20}$ The responses to the MAQ were converted to metabolic equivalent (MET) based on the intensity of the reported activities and expressed as METhours/week. ${ }^{20}$ A standard OGTT was initiated between 0700 and 1100: venous blood specimens for glucose and insulin measurement were obtained before ( 0 minutes) and at 30 minutes and 120 minutes after ingestion of $75 \mathrm{~g}$ flavored glucose (Trutol 75; Custom Laboratories, Baltimore, MD).

\section{Biochemical Measurements}

Plasma glucose was measured with a glucose oxidase method (Yellow Spring Instruments Co., Inc., Yellow Spring, OH). Plasma insulin levels were measured immunochemically in our Endocrine Research Laboratory, using commercial enzymelinked immunosorbent assay kits. HbAlc and fasting plasma lipid profiles were measured in a contract clinical laboratory.

\section{Statistical Analysis}

Data were reported as means \pm SEM. Differences between defined groups were analyzed using unpaired $t$ tests for continuous variables and $\chi^{2}$ test for categoric variables. Interval changes in glycemic, anthropometric, and behavioral measures between the 2 defined testing periods were analyzed using paired $t$ tests. General linear regression models were used to analyze the relationship between temporal changes in anthropometric and metabolic variables. All statistical analyses were performed with the use of SAS statistical software, version 9.3 (SAS Institute Inc., Cary, NC). 
Table 1. Baseline Characteristics of Pathobiology of Prediabetes in a Biracial Cohort Study Participants Who Subsequently Developed Incident Prediabetes or Maintained Normoglycemia (Control) during $\sim 5$ Years of Follow-up*

\begin{tabular}{lccc}
\hline Characteristics & Prediabetes & Control & $P$ Value \\
\hline Number & 73 & 73 & .37 \\
Age $(\mathrm{yr})$ & $48.7 \pm 0.96$ & $48.3 \pm 1.06$ & .85 \\
Women/Men & $46 / 27$ & $48 / 25$ & .84 \\
Black/White & $36 / 37$ & $35 / 38$ & .09 \\
Weight $(\mathrm{kg})$ & $88.7 \pm 2.02$ & $83.5 \pm 2.19$ & .04 \\
BMI $\left(\mathrm{kg} / \mathrm{m}^{2}\right)$ & $31.1 \pm 0.70$ & $29.2 \pm 0.69$ & .02 \\
Waist circumference $(\mathrm{cm})$ & $97.9 \pm 1.34$ & $93.0 \pm 1.71$ & .002 \\
FPG & $94.4 \pm 0.60$ & $91.5 \pm 0.72$ & .05 \\
2 hrPG & $128 \pm 2.80$ & $120 \pm 2.91$ & \\
\hline
\end{tabular}

*Data are mean \pm SEM FPG, fasting plasma glucose; 2 hrPG, plasma glucose value at 2 hours during oral glucose tolerance test. To convert the values for glucose to millimoles per liter, multiply by 0.0555 .

BMI, body mass index; FPG, fasting plasma glucose; PG, plasma glucose.

\section{Results}

\section{Cobort Description}

The characteristics at enrollment of study subjects who developed prediabetes and were so informed during POP-ABC and age- and sex-matched participants who maintained normoglycemia during POP-ABC study (control) are shown in Table 1. As previously reported, ${ }^{17}$ participants who developed incident prediabetes had higher BMI, waist circumference, FPG, and $2 \mathrm{hrPG}$ at enrollment, compared with participants who maintained normoglycemia.

\section{Glycemic, Anthropometric, and Lipidmic Changes}

We assessed the interval changes in plasma glucose and other metabolic endpoints when progressors and nonprogressors to prediabetes during POP$\mathrm{ABC}$ study were rescreened for enrollment in the extension study (PROP-ABC). As already noted, the POP-ABC study was an observational, natural history study that did not entail any drug intervention or lifestyle counseling to alter metabolic endpoints. During the 18-month interval between the end of POP-ABC study and rescreening for the PROP-ABC study, FPG levels decreased by $8.01 \pm$ $1.11 \mathrm{mg} / \mathrm{dL}$ in the prediabetes group and by $2.02 \pm$ $0.64 \mathrm{mg} / \mathrm{dL}$ in the control group $(P<.0001)$. During the same said interval, 2 hrPG levels decreased by $8.21 \pm 3.34 \mathrm{mg} / \mathrm{dL}$ in the prediabetes group but increased by $8.53 \pm 3.17 \mathrm{mg} / \mathrm{dL}$ in the control group $(P=.0004)$ (Figure 2 and Table 2). The corresponding interval changes (prediabetes group vs control group) were $-0.54 \pm 0.72 \mathrm{~kg}$ versus $2.77 \pm 1.25 \mathrm{~kg}(P=.006)$ for body weight and $-1.07 \pm 0.78 \mathrm{~cm}$ versus $1.78 \pm 0.85 \mathrm{~cm}(P=$ $.001)$ for waist circumference (Figure 2 and Table 2). The change in BMI was $0.23 \pm 0.29 \mathrm{~kg} / \mathrm{m}^{2}$ in the prediabetes group and $1.06 \pm 0.46 \mathrm{~kg} / \mathrm{m}^{2}$ in control $(P=.03)$. In the regression analyses, the interval changes in FPG were correlated with interval changes in weight $(\mathrm{r}=0.18, P=.04)$ and waist circumference $(\mathrm{r}=0.20, P=.01)$.

The prediabetes group did not show significant alterations in plasma levels of low-density lipoprotein cholesterol $(111 \pm 3.61 \mathrm{mg} / \mathrm{dL}$ vs $111 \pm 3.79$ $\mathrm{mg} / \mathrm{dL})$, high-density lipoprotein cholesterol (49.2 \pm $2.18 \mathrm{mg} / \mathrm{dL}$ vs $50.5 \pm 1.65 \mathrm{mg} / \mathrm{dL}$ ), or triglycerides $(106 \pm 6.71 \mathrm{mg} / \mathrm{dL}$ vs $102 \pm 5.33 \mathrm{mg} / \mathrm{dL})$ between the end of POP-ABC versus follow-up examination 18 months later, nor did the control group: lowdensity lipoprotein cholesterol, $106 \pm 3.55 \mathrm{mg} / \mathrm{dL}$ versus $106 \pm 3.31 \mathrm{mg} / \mathrm{dL}$; high-density lipoprotein cholesterol, $54.7 \pm 1.73 \mathrm{mg} / \mathrm{dL}$ versus $55.1 \pm 1.57$ $\mathrm{mg} / \mathrm{dL}$; and triglycerides, $91.5 \pm 5.85$ versus $94.2 \pm 4.91$.

\section{Behavioral Changes}

Self-reported FHQ score (lower values indicate healthier eating pattern) decreased from $2.57 \pm$ 0.51 to $2.34 \pm 0.55(-0.23 ; P=.0003)$ in the prediabetes group and from $2.51 \pm 0.50$ to $2.35 \pm$ $0.52(-0.16 ; P=.0005)$ in the control group. The absolute decrease in FHQ score was significantly greater in the prediabetes group versus control group $(-0.23 \pm 0.05$ vs $-0.16 \pm 0.04, P=.002)$. Self-reported MAQ score (higher values indicate greater physical activity) increased nominally from 
Figure 2. Interval changes in glycemia in prediabetes and control groups (A) and within the prediabetes group by ethnicity (B), and interval changes in weight (C) and waist circumference (D) in the prediabetes and control groups. There were no significant ethnic differences in the interval changes in glycemia. $* P=.006,{ }^{*} * P=.001$, $* * * P=.0004, * * * * P<.0001$. To convert the values for glucose to millimoles per liter, multiply by 0.0555 . FPG, fasting plasma glucose; PG, plasma glucose.
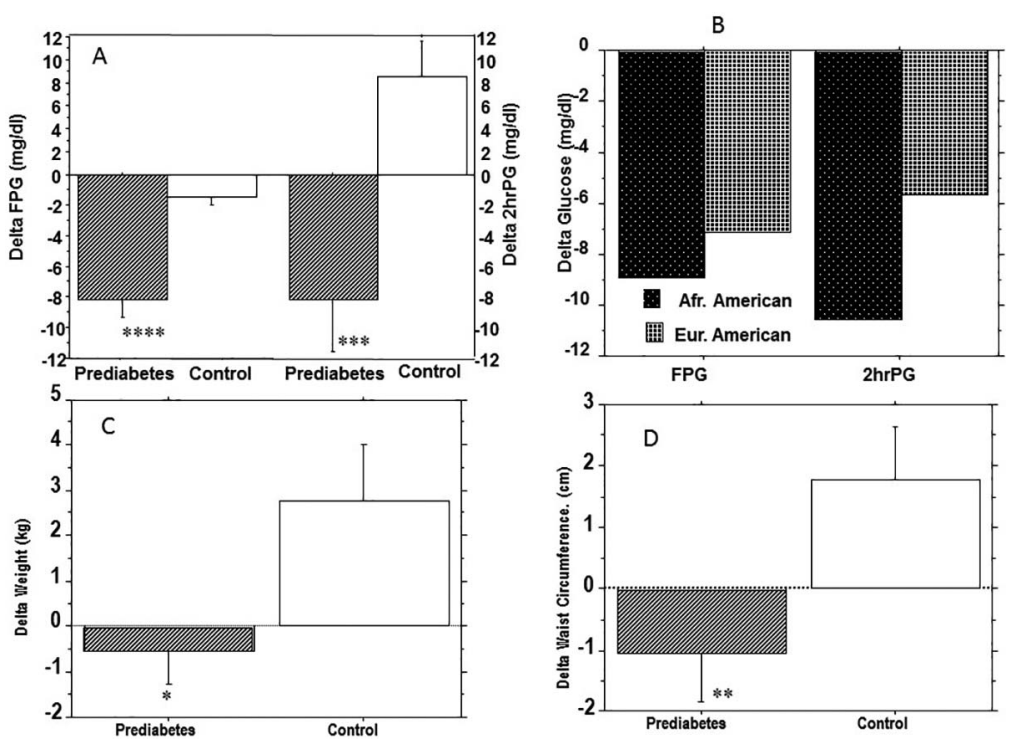

$13.8 \pm 3.03$ MET-hr/wk to $16.5 \pm 2.47$ MET$\mathrm{hr} / \mathrm{wk}$ in the prediabetes group, but decreased nominally from $21.7 \pm 3.39 \mathrm{MET}-\mathrm{hr} / \mathrm{wk}$ to $18.4 \pm$ 2.76 MET-hr/wk in the control group. The absolute change in MAQ score was significantly greater in the prediabetes group versus control group $(2.70 \pm 3.45 \mathrm{MET}-\mathrm{hr} / \mathrm{wk}$ vs $-3.30 \pm 4.20 \mathrm{MET}-$ hr/wk; $P=.002)($ Table 2$)$.

\section{Discussion}

In the present report, we observed that POP-ABC participants who were notified that they had devel-

Table 2. Interval Changes in Glycemic, Anthropometric and Behavioral Measures in Subjects with Incident Prediabetes or Normoglycemia (Control)*

\begin{tabular}{lccc}
\hline Measures & Prediabetes & Control & $P$ Value \\
\hline FPG $(\mathrm{mg} / \mathrm{dL})$ & $-8.01 \pm 1.11$ & $-2.02 \pm 0.64$ & $<.0001$ \\
2 hrPG $(\mathrm{mg} / \mathrm{dL})$ & $-8.21 \pm 3.34$ & $8.53 \pm 3.17$ & .0004 \\
Weight $(\mathrm{kg})$ & $-0.54 \pm 0.72$ & $2.77 \pm 1.25$ & .006 \\
BMI $\left(\mathrm{kg} / \mathrm{m}^{2}\right)$ & $0.23 \pm 0.29$ & $1.06 \pm 0.46$ & .03 \\
Waist $(\mathrm{cm})$ & $-1.07 \pm 0.78$ & $1.78 \pm 0.85$ & .001 \\
FHQ score & $-0.23 \pm 0.05$ & $-0.16 \pm 0.04$ & .002 \\
MAQ (MET-hr/wk) & $2.70 \pm 3.45$ & $-3.30 \pm 4.20$ & .03 \\
\hline
\end{tabular}

*Data are mean \pm SEM FPG, fasting plasma glucose; 2 hrPG, plasma glucose value at 2 hours during oral glucose tolerance test. To convert the values for glucose to millimoles per liter, multiply by 0.0555 .

BMI, body mass index; FHQ, Food Habits Questionnaire; FPG, fasting plasma glucose; MAQ, Modifiable Activity Questionnaire; MET, metabolic equivalent; PG, plasma glucose. 
no therapeutic intervention was offered to the participants who progressed to prediabetes. However, a written report of the confirmatory OGTT results indicating the development of IFG and/or IGT was provided to participants reaching those endpoints. Thus, our present findings suggest that the mere notification of incident prediabetes status may trigger beneficial changes in glycemia and body size in AAs and EAs with parental history of T2DM.

The mechanism(s) for the observed benefits in glycemia, glucose tolerance, and adiposity following notification of incident prediabetes status are unclear but could have involved self-directed lifestyle modification. To explore such a mechanism, we analyzed changes in self-reported dietary and physical activity behaviors in the 2 comparison groups of participants across the 18-month interval between conclusion of the POP-ABC study and initiation of the PROP-ABC study. We found that FHQ scores decreased in both the prediabetes and control groups (indicating healthier eating patterns), but the improvement was significantly greater in the prediabetes group. Similarly, participants in the prediabetes group reported a significant $6 \mathrm{MET}-\mathrm{hr} / \mathrm{wk}$ greater physical activity than did those in the control group. These behavioral alterations, if sustained, could explain the glycemic and weight benefits observed in the prediabetes group. In addition to self-directed behavioral changes, it is possible that some participants may have discussed their prediabetes status with their primary care providers and may have received lifestyle counseling outside our study.

Did the Hawthorne effect or regression to the mean play a role in our findings? Subjects enrolled in research studies may alter their behavior and produce unintended results, from the increased attention and encounters associated with research participation (Hawthorne effect). ${ }^{21}$ Usually, the Hawthorne effect dissipates within approximately 4 months or once study procedures cease. ${ }^{22,23}$ Notably, our POP-ABC study had a follow-up period of 5.5 years (mean, 2.62 years) and the interval between end of study and retesting of participants was 18 months. Thus, it is unlikely that our present findings could be explained by the Hawthorne effect. Moreover, there is no reason why any Hawthorne effect would be restricted to the prediabetes group. The statistical phenomenon of regression to the mean describes the tendency of individuals with outlier values on a given measure to have sponta- neously lower values on retesting, without any intervention. The use of contemporaneous measurements in a matched control group, as was done in the present study, usually obviates the risk of misinterpretation due to regression to the mean. ${ }^{24,25}$

Two previous national surveys had reported discordant findings on the impact of awareness of prediabetes status on health behavior. ${ }^{14,15}$ Data from the 2006 National Health Interview Survey showed that of the $\sim 4 \%$ of US adults who had been told that they had prediabetes, $68 \%$ reported active attempts to lose or control weight, 55\% reported increased physical activity, $60 \%$ reported less fat consumption, and $42 \%$ reported trying all 3 approaches. ${ }^{14}$ In a different report, investigators analyzed data on 24-hour dietary recall, self-reported diabetes and prediabetes awareness status, and FPG and HbA1c values from the 2005 to 2010 National Health and Nutrition Examination Survey. ${ }^{15}$ Persons unaware of diabetes and prediabetes were identified by FPG $<126 \mathrm{mg} / \mathrm{dL}$ or $\mathrm{HbA1c}$ $<6.5 \%$ and FPG $100 \mathrm{mg} / \mathrm{dL}$ to $125 \mathrm{mg} / \mathrm{dL}$ or HbA1c of $5.7-6.4 \%$, respectively. ${ }^{15}$ People with diagnosed diabetes reported consumption of less carbohydrates and more protein compared with those with undiagnosed diabetes. ${ }^{15}$ However, the authors observed no significant differences in macronutrient intake by awareness of prediabetes status. ${ }^{15}$ The conclusion from the 2005 to $2010 \mathrm{Na}$ tional Health and Nutrition Examination Survey data was that knowledge of glycemic status induced healthier dietary patterns for people with diabetes but not those with prediabetes. Unlike the crosssectional surveys based on self-reported prediabetes status, our prospective study used a rigorous ascertainment of incident prediabetes status, using OGTT and independent adjudication. Thus, the mechanism of becoming aware of incident prediabetes in the POP-ABC study was the documentation that a participant's FPG and/or 2hrPG values had drifted upward from normal glucose regulation to prediabetes (IFG and/or IGT). Our findings support a beneficial impact of prediabetes awareness on health behavior and metabolic endpoints. We argue that awareness of incident prediabetes status through direct communication of measured glucose values, as was done in the POP-ABC study, was impactful in triggering behavioral change.

Remarkably, the vast majority of people with prediabetes in the general primary care population remain undiagnosed and unaware of their condi- 
tion. ${ }^{2,14,15,26}$ In the 2006 National Health Interview Survey, only an estimated $4 \%$ of US adults had been told they had prediabetes. ${ }^{15}$ Although $\sim 34 \%$ of US adults had prediabetes in 2015 , only $11.6 \%$ self-reported that they had been diagnosed with prediabetes by a health care worker. ${ }^{2}$ Awareness of and response to prediabetes among health care providers is even much lower. Analyzing HbA1c data from the 2012 National Ambulatory Medical Care Survey that targeted adults aged $>45$ years without diagnosed diabetes who had an HbA1c test within 90 days $(\mathrm{N}=11,167,004$ weighted visits), the prevalence of prediabetes was found to be $33.6 \% .{ }^{26}$ However, $<1 \%$ of patients with $\mathrm{HbA} 1 \mathrm{c}$ results consistent with prediabetes had formal diagnosis and documentation of prediabetes in the medical records. ${ }^{26}$ Clearly, there is need for a greater awareness and early action regarding the diagnosis and management of prediabetes. Once a diagnosis is obtained, using FPG, OGTT, or HbA1c values, the clinical significance of prediabetes needs to be communicated clearly to all affected patients (6 to 8 ), as the present report has shown that notification of prediabetes status (even without active intervention) may trigger beneficial self-directed lifestyle modifications. In addition, current guidelines by the ADA recommend referring people with prediabetes for intensive lifestyle modification. $^{4}$

All authors participated in the interpretation of the studies and review of the manuscript. SD-J, as principal investigator and guarantor of this manuscript, designed the study, analyzed data, and wrote the manuscript. IO performed statistical analysis, reviewed, and revised manuscript; and NU collected data, reviewed, and revised the manuscript. FC collected data, reviewed, and revised the manuscript.

We are indebted to the participants who volunteered for this study.

To see this article online, please go to: bttp://jabfm.org/content/ 32/1/20.full.

\section{References}

1. American Diabetes Association and National Institute of Diabetes, Digestive and Kidney Diseases. The prevention or delay of type 2 diabetes. Diabetes Care 2002;25:742-9.

2. Centers for Disease Control and Prevention. National Diabetes Statistics Report, 2017. Atlanta, GA: Centers for Disease Control and Prevention, US Dept of Health and Human Services; 2017. Available from: https://www.cdc.gov/diabetes/data/statistics/ statistics-report.html. Accessed August 2018.
3. Ogurtsova K, da Rocha Fernandes JD, Huang Y, et al. IDF Diabetes Atlas: global estimates for the prevalence of diabetes for 2015 and 2040. Diabetes Res Clin Pract 2017;128:40-50.

4. American Diabetes Association. Standards of medical care in diabetes-2018. Diabetes Care 2018;41: S1-S155.

5. World Health Organization. Diabetes mellitus: report of a WHO Study Group. Tech. Rep. Ser. 1985, no. 727. Geneva, World Health Organization; 1985.

6. Nathan DM, Davidson MB, DeFronzo RA, et al. Impaired fasting glucose and impaired glucose tolerance: implications for care. Diabetes Care 2007;30: 753-9.

7. Brannick B, Wynn A, Dagogo-Jack S. Prediabetes as a toxic environment for the initiation of microvascular and macrovascular complications. Exp Biol Med (Maywood) 2016;241:1323-31.

8. Brannick B, Dagogo-Jack S. Prediabetes and cardiovascular disease: pathophysiology and interventions for prevention and risk reduction. Endocrinol Metab Clin North Am 2018;47:33-50.

9. Knowler WC, Barrett-Connor E, Fowler SE, et al. Reduction in the incidence of type 2 diabetes with lifestyle intervention or metformin. N Engl J Med 2002;346:393-403.

10. Li G, Zhang P, Wang J, et al. Cardiovascular mortality, all-cause mortality, and diabetes incidence after lifestyle intervention for people with impaired glucose tolerance in the Da Qing Diabetes Prevention Study: a 23-year follow-up study. Lancet Diabetes Endocrinol 2014;2:474-80.

11. Lindström J, Ilanne-Parikka P, Peltonen M, Aunola $\mathrm{S}$, Eriksson JG, Hemiö K. Sustained reduction in the incidence of type 2 diabetes by lifestyle intervention: follow-up of the Finnish Diabetes Prevention Study. Lancet 2006;368:1673-9.

12. Perreault L, Pan Q, Mather KJ, et al. Effect of regression from prediabetes to normal glucose regulation on long-term reduction in diabetes risk: results from the Diabetes Prevention Program Outcomes Study. Lancet 2012;379:2243-51.

13. Diabetes Prevention Program Research Group. Achieving weight and activity goals among Diabetes Prevention Program lifestyle participants. Obes Res 2004; $12: 1426-34$.

14. Centers for Disease Control and Prevention. Selfreported prediabetes and risk-reduction activitiesUnited States, 2006. MMWR Morb Mortal Wkly Rep 2008;57:1203-5.

15. Bardenheier BH, Cogswell ME, Gregg EW, Williams DE, Zhang Z, Geiss LS. Does knowing one's elevated glycemic status make a difference in macronutrient intake? Diabetes Care 2014;37:3143-9.

16. Dagogo-Jack S, Edeoga E, Ebenibo S, ChappJumbo E. Pathobiology of prediabetes in a bi-racial cohort (POP-ABC) study: baseline characteristics of 
enrolled subjects. J Clin Endocrinol Metab 2013;98: 120-8.

17. Dagogo-Jack S, Edeoga C, Ebenibo S, Nyenwe E, Wan J; for the Pathobiology of Prediabetes in a Biracial Cohort (POP-ABC) Research Group. Lack of racial disparity in incident prediabetes and glycemic progression among black and white offspring of parents with type 2 diabetes: the Pathobiology of Prediabetes in a Biracial Cohort (POP-ABC) Study. J Clin Endocrinol Metab 2014;99:E1078-87.

18. Krista AR, Shattuck AL, Henry HJ. Patterns of dietary behavior associated with selecting diets low in fat: reliability and validity of a behavioral approach to dietary assessment. J Am Diet Assoc 1990;90:214-20.

19. Kriska AM, Knowler WC, LaPorte RE, et al. Development of questionnaire to examine relationship of physical activity and diabetes in Pima Indians. Diabetes Care 1990;13:401-11.

20. Boucher AB, Adesanya EAO, Owei I, et al. Dietary habits and leisure-time physical activity in relation to adiposity, dyslipidemia, and incident dysglycemia in the Pathobiology of Prediabetes in A Biracial Cohort Study. Metabolism 2015;64:1060-7.
21. Parsons HM. What Happened at Hawthorne?: new evidence suggests the Hawthorne effect resulted from operant reinforcement contingencies. Science 1974;183:922-1932.

22. Cizza G, Piaggi P, Rother KI, Csako G; Sleep Extension Study Group. Hawthorne effect with transient behavioral and biochemical changes in a randomized controlled sleep extension trial of chronically shortsleeping obese adults: implications for the design and interpretation of clinical studies. PLoS One 2014 Aug 20;9:e104176.

23. McCambridge J, Witton J, Elbourne DR. Systematic review of the Hawthorne effect: new concepts are needed to study research participation effects. J Clin Epidemiol 2014;3:267-77.

24. $\mathrm{Yu} \mathrm{R}$, Chen L. The need to control for regression to the mean in social psychology studies. Front Psychol 2015;5:1574.

25. Linden A. Assessing regression to the mean effects in health care initiatives. BMC Med Res Methodol 2013;13:119.

26. Mainous AG, Tanner RJ, Baker R. Prediabetes diagnosis and treatment in primary care. J Am Board Fam Med 2016;29:283-5. 